\title{
Dor, função pulmonar e força muscular respiratória no pré e pós-operatório de mulher mastectomizada
}

\author{
Pain, pulmonary function and \\ respiratory muscle strength in pre-and \\ postoperative woman mastectomized
}

FisiSenectus. Unochapecó Ano 4, n. 1 - Jan/Jun. 2016 p. $32-41$

Ana Laura Nicoletti Carvalho Petry. ana.petry@uffs.edu.br

Doutora em Ciências pela Universidade de São Paulo (USP).Fisioterapeuta na Universidade Federal da Fronteira Sul (UFFS) - Campus Passo Fundo/RS.

Manoele Molossi Bernardi. manoelem.bernardi@yahoo.com.br Fisioterapeuta formada pela Universidade Regional Integrada do Alto Uruguai e das Missões (URI) - Campus Erechim/RS.

Ana Lucia Bernardo de Carvalho Morsch. analuepm@yahoo.com.br Mestre em Ciências pela Universidade Federal de São Paulo (UNIFESP). Docente do Curso de Fisioterapia na Universidade Regional Integrada do Alto Uruguai e das Missões (URI) - Campus Erechim/RS.

\section{Resumo}

Introdução: 0 câncer de mama é a neoplasia que mais atinge as mulheres brasileiras. A cirurgia é o tratamento mais indicado e no pós-operatório a fisioterapia pode atuar para prevenir possíveis complicações. Objetivos: Verificar os efeitos da fisioterapia na dor pósoperatória, na função pulmonar e na força muscular respiratória em uma mulher no pósoperatório de câncer de mama nos momentos pré e pós-procedimento cirúrgico. Materiais e métodos: Indivíduo, sexo feminino, 61 anos, que realizou mastectomia radical. Foram analisadas as forças musculares respiratórias; a função pulmonar e a dor na mama. Todas as avaliações foram conduzidas nos momentos de avaliação inicial, pós-operatório imediato e pós-execução do protocolo de fisioterapia. O protocolo constou de exercícios específicos para os primeiros quinze dias de pós-operatório que objetivaram alcançar até $90^{\circ}$ de amplitude de movimento e outros após quinze dias de pós-operatório com a amplitude de movimento completa (duas séries de 10 repetições de cada exercício). Conclusão: Em relação à dor na mama observou-se um aumento no pós-operatório, reduzindo gradualmente no decorrer das sessões; já a força muscular respiratória e a função pulmonar encontravam-se acima dos valores previstos no pré-operatório, reduzindo no pós-operatório e retornando aos valores esperados (previstos) após as sessões fisioterapêuticas. Os exercícios contribuíram para normalização dos parâmetros respiratórios analisados e melhora da dor na mama, contudo eles não foram capazes de retornar os parâmetros respiratórios aos encontrados no pré-operatório. Entendemos ser importante um tratamento fisioterapêutico precoce em mulheres que realizaram mastectomia, para evitar possíveis complicações e para uma recuperação mais rápida e eficaz.

\section{Palavras-chave}

Neoplasias da mama; Reabilitação; Dor pós-operatória;

Testes respiratórios.

\section{Fisi'enectus}




\begin{abstract}
Backgroud: Breast cancer is a cancer that affects Brazilian women. Surgery is the best treatment and postoperative physical therapy may act to prevent possible complications. Objective: To assess the effects of physical therapy in postoperative pain, pulmonary function, as well as respiratory muscle strength in a woman in the post-operative breast cancer in pre- and post-surgical procedure. Materials and methods: Individual, female, 61, who underwent radical mastectomy. Respiratory muscle strength were analyzed; as well as pulmonary function and pain in the breast. All evaluations were conducted in times of initial evaluation, postoperative and post-implementation of the physiotherapy protocol. The protocol included specific exercises for the first two weeks after surgery that aimed to reach up to $90^{\circ}$ range of motion and other after fifteen days postoperatively with the full range of motion (two sets of 10 repetitions of each exercise). Conclusions: In relation to breast pain, there was an increase in the postoperative period, reducing gradually over the course of the sessions; have respiratory muscle strength and lung function were above the values predicted preoperatively, reducing postoperative and returning the expected values (provided) after the physical therapy sessions. The exercises contributed to normalization of respiratory parameters analyzed and improvement of breast pain, yet they were not able to return the respiratory parameters to found preoperatively. We believe is important early physical therapy in women who underwent mastectomy to avoid possible complications and a faster and more effective recovery.
\end{abstract}

\title{
Keywords
}

Breast neoplasms; Rehabilitation; Pain postoperative; Breath tests.

\section{Introdução}

$\infty \times \infty \times \infty \times \infty \times \infty \times \infty \times \infty \times \infty \times \infty \times \infty \times \infty \times \infty \times \infty \times$

O câncer de mama é uma doença maligna, caracterizado por um desordenado crescimento celular. Compromete mulheres em países desenvolvidos ocorrendo geralmente acima dos quarenta anos de idade. É o segundo tipo de câncer com maior frequência no mundo, e sua incidência vem aumentando a cada ano ${ }^{1}$. É o câncer mais temido pelas mulheres, pois suas consequências psicológicas em relação à sexualidade e a percepção da imagem pessoal são graves. Seu tratamento inclui a intervenção cirúrgica que pode ser de vários tipos, quimioterapia, hormonioterapia, radioterapia e fisioterapia ${ }^{2}$.

A cirurgia é o tratamento mais indicado e independente do tipo de mastectomia o pós-operatório pode trazer muitas complicações como aderência pericicatricial, restrição da amplitude de movimento, deiscência cicatricial, dor, linfedema ${ }^{3}$ e comprometimento do sistema respiratório².

Os comprometimentos da força muscular respiratória e da função pulmonar estão, provavelmente, intimamente relacionados com a limitação no movimento do membro superior afetado e com as complicações cirúrgicas no momento pós-operatório ${ }^{2,3}$.
Entende-se que exercícios cinesioterapêuticos específicos para os membros superiores (afetado e não afetado) podem, por si só, atuar na recuperação da cinética do sistema respiratório em mastectomizadas. Portanto, este trabalho objetivou a aplicação de um protocolo de exercícios cinesioterapêuticos para membros os superiores, com a finalidade de verificar os efeitos da fisioterapia na dor pós-operatória, na função pulmonar e na força muscular respiratória em uma mulher no pós-operatório de câncer de mama nos momentos pré e pós-procedimento cirúrgico e após as 10 sessões de fisioterapia.

\section{Materiais e métodos}

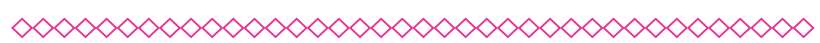

0 presente estudo caracterizou-se como um estudo de caso do tipo descritivo de caráter quantitativo e cunho longitudinal. A amostra foi composta de uma mulher com câncer de mama à direita que realizou o procedimento cirúrgico para retirada total da mama. A escolha da participante da pesquisa foi a partir da lista de agendamentos para a realização do procedimento cirúrgico médico na Fundação Hospitalar Santa Terezinha de Erechim/RS. A paciente aceitou participar deste 
estudo e assinou o Termo de Consentimento Livre e Esclarecido.

No contato inicial pré-operatório foi realizada a avaliação fisioterapêutica. A avaliação constava de uma ficha de avaliação contendo dados pessoais gerais e dados referentes à história da doença. Após, foi realizada a avaliação da intensidade da dor por meio da aplicação da Escala Visual Analógica (EVA). Para essa avaliação a paciente foi questionada quanto ao seu grau de dor sendo que "0" significa ausência total de dor e "10" o nível de dor máxima suportável pela paciente. A partir dessas informações iniciais, a paciente indicou a intensidade de sua dor na escala.

Após, a paciente foi submetida aos testes respiratórios. Primeiramente, foi realizada a espirometria com aparelho Micromedical, modelo Espirolab II, para avaliação da função pulmonar, com valores conforme a Sociedade Brasileira de Pneumologia e Tisiologia. Com a paciente sentada em uma cadeira, com os braços ao longo do corpo e membros inferiores a $90^{\circ}$ de flexão, mantendo o nariz ocluído foi solicitado que ela inspirasse profundamente até encher plenamente os pulmões e, em seguida realizasse uma expiração rápida, soprando todo o volume de ar contido nos pulmões. Esta expiração rápida e forçada foi mantida pelo maior tempo possível. Dessa forma, foi realizado por três vezes o teste, sendo que estes foram reprodutíveis e aceitáveis.

Secundariamente, a paciente foi submetida à manovacuometria para obtenção dos valores das pressões respiratórias máximas inspiratórias (PImáx) e expiratórias (PEmáx) que seguiu as recomendações das Diretrizes das Provas de Função Pulmonar ${ }^{4}$. Foi utilizado um manovacuômetro analógico e bocais, traqueias e clipe nasal de plástico, ambos esterilizados. A PImáx que é a maior pressão gerada durante uma inspiração forçada contra uma via aérea ocluída, e foi realizada a partir do esforço inspiratório iniciado ao nível do volume residual (volume de gás contido nos pulmões, após uma expiração máxima). A paciente expirou até alcançar seu volume residual, imediatamente conectou a boca ao bocal e efetuou um esforço inspiratório máximo, mantendo-o por pelo menos dois segundos. O comando verbal utilizado para facilitar a execução dessa manobra foi: "solte todo o ar e depois puxe o ar com toda força no bocal" ${ }^{\prime 5}$.
Para mensurar a PEmáx que é a maior pressão que pode ser desenvolvida durante um esforço expiratório vigoroso contra uma via aérea ocluída, foi realizada durante o esforço expiratório iniciado ao nível da capacidade pulmonar total (volume de gás contido nos pulmões após uma inspiração máxima $)^{5}$. Solicitou-se que a mesma inspirasse até alcançar sua capacidade pulmonar total e em seguida conectou-se a boca ao bocal e efetuou um esforço expiratório máximo. 0 comando verbal utilizado para facilitar a execução dessa manobra foi: "puxe o ar até encher todo pulmão e depois sopre com toda força no bocal". O tempo expiratório alcançado foi mantido por um a três segundos, pois períodos mais prolongados podem causar síncopes devido à redução do debito cardíaco causado pela elevação da pressão intratorácica. A paciente realizou cinco manobras aceitáveis e reprodutíveis máximas (isto é, diferenças de 10\% ou menos entre os valores): o valor registrado foi o mais elevado.

No $2^{\circ}$ dia de pós-operatório realizou-se novamente as avaliações de força muscular respiratória, função pulmonar e avaliação da intensidade da dor por meio da manovacuometria, da espirometria e a da EVA, respectivamente. Após esta primeira reavaliação, iniciou-se o protocolo de exercícios cinesioterapêuticos. O protocolo cinesioterapêutico constou de exercícios preconizados por Camargo e Marx ${ }^{5}$. Até o $15^{\circ}$ dia de pós-operatório foram realizados exercícios de adução e abdução das escápulas e dos ombros seguido de relaxamento; flexão anterior e abdução do braço à $90^{\circ}$; rotação interna e externa do ombro; elevação simultânea e relaxamento dos ombros; inclinação da cabeça; rotação da cabeça e elevação alternada dos ombros.

Após o $15^{\circ}$ dia de pós-operatório forma realizados os seguintes exercícios: (1) "Mãos apoiadas sobre o queixo, cotovelos abertos, colocar as mãos sobre a cabeça e a partir daí elevação dos ombros"; (2) "Extensão total dos braços, após apoiar mãos sobre a cabeça e inclinar braços alternadamente"; (3) "Mãos sobre a cabeça, elevar lentamente até extensão total do braço"; (4) Na posição de extensão total do braço "baixar alternadamente um braço e depois o outro"; (5) "Mãos apoiadas atrás da nuca, cotovelos abertos, fechá-los e abri-los"; (6) "Mãos apoiadas sobre o queixo, extensão anterior dos braços e flexão com abertura dos cotovelos 
e palmas das mãos viradas para fora"; (7) "Mãos atrás da nuca, cotovelos abertos, abdução e flexão lateral do tronco"; (8) "Inclinação do tronco, abdução do braço com mãos entrelaçadas"; (9) "Flexão anterior total dos braços, levar a toalha atrás da nuca com flexão de cotovelos e em abdução do braço" e (10)"Flexão anterior total dos braços e inclinações laterais" ${ }^{\prime \prime}$. 0 protocolo de exercícios foi realizado por cinco semanas, com duas sessões semanais, totalizando dez sessões.

Após o término 10 sessões de tratamento fisioterapêutico realizou-se a reavaliação final contando com os testes de espirometria e da manovacuometria. Salienta-se que a avaliação da intensidade da dor por meio da escala EVA foi realizada em todos os atendimentos sempre no final da sessão.

A análise dos dados foi conduzida por meio da utilização do programa SPSS 15.0 (SPSS Inc., Chicago, ILL, USA). Os dados foram apresentados de forma descritiva simples, onde as variáveis quantitativas foram apresentadas por meio de frequências relativas (percentuais). Os resultados para força muscular respiratória, função pulmonar e avaliação da dor nos momentos pré, pós-operatório imediato e pós-operatório tardio (ao final de todas as intervenções) foram expressos em média e desvio padrão.

0 presente trabalho seguiu os princípios éticos contidos na Declaração de Helsique, sendo aprovado pelo Comitê de Ética em pesquisa da URI Campus de Erechim/RS sob o número da CAAE 08756712.8.0000.5351.

\section{Resultados}

$\infty \times \infty \times \infty \times \infty \times \infty \times \infty \times \infty \times \infty \times \infty \times \infty \times \infty \times \infty \infty \infty$

A participante do estudo se tratava de uma mulher, de 60 anos de idade, com 1,67 m de altura e $78 \mathrm{Kg}$, aposentada, procedente de Erechim/ RS, tendo como médico responsável Fábio Pais. A participante foi diagnosticada com neoplasia da mama à direita, apresentava hipotireoidismo e hipertensão arterial sistêmica e não tinha antecedentes de tabagismo ou etilismo. Em relação à sua história familiar de patologias, a mesma relata a presença de câncer de sistema nervoso central (irmã) e câncer no esôfago (sobrinha).
Em relação à dor referida na mama, no pré-operatório a paciente relatou que a mesma se apresentava de intensidade leve $(E V A=3)$. Já no pós-operatório imediato observou-se um aumento do quadro álgico para intensidade severa (EVA=8), porém no decorrer das 10 sessões fisioterapêuticas observou-se uma diminuição gradual do quadro álgico para (EVA=1), sendo considerada uma dor leve. 0 protocolo de exercícios cinesioterapêuticos precoce pode ter contribuído na redução do quadro álgico (Figura 1).

No que se diz respeito à força da musculatura respiratória avaliada por meio da manovacuometria, observou-se que no pré-operatório a mesma encontrava-se acima dos valores previstos para o sexo e para idade ${ }^{6}$, reduzindo os valores no pós-operatório e retornando aos valores esperados (previstos) após os 10 atendimentos fisioterapêuticos. Porém, observou-se que o reestabelecimento dos valores das pressões respiratórias não alcançaram os valores pré-operatórios. Verificou-se que os exercícios propostos podem ter colaborado para o reestabelecimento da força muscular respiratória prevista para esta paciente, não sendo, no entanto, suficientes para alcançar os valores encontrados no pré-operatório que superaram os valores previstos (Tabela $\mathbf{1}$ ).

Em relação à função pulmonar avaliada por meio da espirometria, pode-se observar que os valores pré-operatórios encontravam-se dentro dos valores previstos, reduzindo no pós-operatório e retornando praticamente aos valores previstos após os 10 atendimentos fisioterapêuticos. No entanto, os exercícios propostos não foram suficientes, por si só, para o reestabelecimento dos valores encontrados no pré-operatório (Tabela 2).

\section{Discussão}

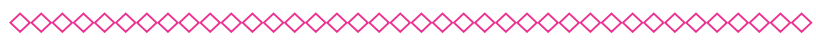

Existem várias técnicas de intervenção cirúrgica e a escolha irá depender do caso de cada paciente. Dentre elas podemos citar as cirurgias conservadoras que incluem a quadrantectomia e a tumorectomia e as cirurgias radicais denominadas mastectomias. Independente da abordagem cirúrgica, radical ou conservadora, as complicações físicas e motoras podem aparecer imediatamente 
ou tardiamente à cirurgia, sendo a dor uma das principais ${ }^{7}$.

A dor é descrita como uma experiência emocional e sensorial desagradável que está associada a uma lesão tecidual. Ela é classificada através de vários critérios como nociceptiva (somática ou visceral), neuropática (nervos periféricos, raiz nervosa e sistema nervoso central), duração (aguda ou crônica) com causas oncológicas, traumáticas ou pós-operatórias ${ }^{8}$. Quando foram avaliadas cinco mulheres submetidas à mastectomia radical modificada e a quadrantectomia as mesmas relataram como queixa principal dor (40\%) e edema $(40 \%)^{9}$.

Dos doentes oncológicos, 35 a 45\% sentem dor tanto no dia do diagnóstico como na fase mais tardia da doença. Esta dor geralmente está associada com a neoplasia devido à invasão ou a compressão das estruturas, devido ao tratamento que inclui a radioterapia, fibrose, neuropatia, quimioterapia e o pós-operatório ${ }^{3}$. A dor também pode estar associada à presença do dreno aspirativo ${ }^{10}$. No caso deste estudo, a paciente permaneceu com dreno durante 20 dias de pós-operatório.

Considerando a paciente estudada, a dor inicial foi considerada aguda e de origem oncológica devido à presença do tumor de mama. No pré-operatório, a mesma referia dor leve e um aumento do quadro álgico no momento pós-operatório imediato. Acredita-se que o próprio procedimento cirúrgico, ou seja, o tipo de cirurgia (mastectomia radical), a incisão, a presença de dreno e/ou a manipulação intraoperatória dos tecidos sugerem esta elevação da escala de dor após a cirurgia.

As mulheres que realizam a fisioterapia no pós-operatório tardio apresentam dor, demostrando a necessidade de uma intervenção fisioterapêutica o mais precoce possível ${ }^{11}$. Os pacientes que iniciam a fisioterapia em períodos diferidos no tempo após a cirurgia apresentam complicações resultantes da mesma, sendo que o seu grau de gravidade, maior ou menor, parece estar relacionado com o tempo de início da fisioterapia, assim como o sucesso do seu tratamento. Para estes autores a fisioterapia deve ser iniciada imediatamente no pós-operatório, mais precisamente 24 horas após a cirurgia ${ }^{12}$. Neste estudo foi realizada uma intervenção precoce objetivando evitar ou minimizar as complicações no pós-operatório tardio.
Na literatura existem muitos programas de reabilitação para mulheres no pós-operatório de mama. Alguns programas relatam a aplicação de contrações isométricas da musculatura do ombro e braço, nos quais a paciente deve levantar os braços com as mãos unidas em flexão, abdução e rotação do ombro até o limiar de dor; outros preconizam a realização de exercícios ativo-livres em todos os movimentos normais do ombro; alguns empregam alongamentos e fortalecimentos juntamente com exercícios para a cabeça, pescoço tronco e membros $^{13}$. Ainda, não existe um consenso sobre qual deve ser a amplitude de movimento adequada para os membros superiores para a realização de exercícios no pós-operatório ${ }^{13}$. Alguns autores sugerem que o movimento não deva passar dos $40^{\circ}$ de flexão e abdução no primeiro e segundo dia de pós-operatório, $45^{\circ}$ no terceiro dia, de $45^{\circ}$ a $90^{\circ}$ de flexão e $45^{\circ}$ de abdução no quarto dia, e livre após 7 dias. Outros autores relatam que não deve existir movimento do ombro durante 5 dias $^{13}$.

0 protocolo utilizado neste estudo está de acordo com o descrito por Camargo e Marx ${ }^{5}$, pois os mesmos afirmam que é de extrema importância que a fisioterapia seja realizada com técnicas específicas para eliminar as complicações. A cinesioterapia realizada ativamente será decisiva na reabilitação da amplitude de movimento e também na redução dessas complicações. Porém, se a cinesioterapia for realizada com uma amplitude máxima, ela ajudará na recuperação do membro superior sendo responsável também pela maior quantidade de líquido drenado e também pela duração de permanência do dreno. Estes exercícios em amplitude máxima poderão favorecer a separação das bordas cirúrgicas, diminuir o processo normal de cicatrização e aumentar o risco de infecções. A amplitude de $90^{\circ}$ de abdução e flexão são muito eficientes para a rápida retomada da função do ombro, para prevenir aderências e retrações e evitar outras complicações ${ }^{5}$.

Ainda para os mesmos autores ${ }^{5}$, os exercícios do pós-operatório em até quinze dias deverão ser em amplitudes limitadas, para que não haja tração da incisão e da pele. Inicia-se com exercícios posturais simples, exercícios dinâmicos (respiratórios, membros superiores e cervical) e exercícios globais menos específicos. Dos quinze dias em diante, realiza-se de fato a reabilitação funcional ativa. 
Os exercícios deverão ser realizados na amplitude máxima em todos os eixos de movimento. 0 ganho de amplitude deve ser gradual e de preferencialmente com ausência de dor aguda. Nesta fase os exercícios deverão ser variados, desde deitados até em pé.

Além desses aspectos, sabe-se que mesmo que a cirurgia da mama não invada o tórax, as pacientes podem ter complicações pulmonares, devido, à diminuição da mobilidade, por isto a mobilização precoce torna-se importante para evitar ou eliminar as complicações respiratórias. A presença do dreno leva a uma diminuição da expansibilidade pulmonar, favorecendo essas complicações ${ }^{10}$.

A avaliação das pressões respiratórias máximas nos fornece valores força muscular respiratória. A PImáx consiste em um índice da força dos músculos inspiratórios (diafragma e intercostais externos), enquanto a PEmáx mede a força dos músculos expiratórios (abdominais e intercostais internos). Vale ressaltar que as medidas da PImáx são de maior importância clínica devido aos músculos inspiratórios suportarem grandes cargas de trabalho ventilatório. As mensurações da PEmáx são importantes para diferenciar fraqueza neuromuscular de músculos abdominais e uma fraqueza específica do diafragma ${ }^{14}$.

Os valores previstos por meio das equações preditivas descrita pelos autores ${ }^{6}$ para esta paciente de acordo com sua idade foram - $81 \mathrm{cmH}_{2} \mathrm{O}$ para PImáx e $79 \mathrm{cmH}_{2} \mathrm{O}$ para PEmáx. Nos resultados obtidos pela paciente mostra que no momento pré-operatório a paciente apresentou valores que superam os preditos tanto de PImáx quanto de PEmáx, ocorrendo uma diminuição das mensurações no pós-operatório e retornando aos valores preditos após as 10 sessões de fisioterapia. No entanto, esses valores de pós-operatório tardio após os exercícios não retornaram aos valores pré-operatórios para as forças musculares respiratórias. Este achado nos permite pensar que os exercícios podem colaborar para obter retorno aos valores iniciais das forças musculares respiratórias, não sendo suficientes para o retorno completo aos valores pré-operatórios.

Embora os exercícios realizados melhorarem a mobilidade do membro superior, que está indiretamente ligada à mobilidade torácica, eles não foram específicos para treinamento da musculatura respiratória. Também se hipotetiza que esses valores não retornaram aos do pré-operatório devido ao pouco tempo de tratamento, pois foram apenas 10 sessões. Faz-se necessária a realização de um protocolo de exercícios de longa duração para uma melhor comparação.

Em relação à função pulmonar, existem muitos estudos que objetivam a validação de índices para a predição do risco cirúrgico como forma de prevenir a ocorrência de complicações respiratórias. A utilização da espirometria só é eficaz naqueles pacientes que realizarão ressecção pulmonar ou que tenham algum problema respiratório ${ }^{15}$.

Nosso estudo discorda de Bellinetti e Thomson $^{15}$, uma vez que a espirometria pode ser muito útil nas cirurgias de mama, pois nos fornece informações dos valores basais da função pulmonar e de como pode ocorrer alteração da função pulmonar após o ato cirúrgico. A opção de tratamento fisioterapêutico precoce proposto neste trabalho pode ter contribuído para retorno da função pulmonar aos valores previstos. É importante salientar que não é possível afirmar que o retorno dos valores da função pulmonar ao previsto se deva exclusivamente ao tratamento fisioterapêutico precoce ou se a normalização dos valores da função pulmonar ocorreria também sem nenhuma intervenção em função apenas do tempo pós-operatório. Um porém deste estudo foi a falta de participantes para a realização de um grupo controle (sem intervenção fisioterapêutica), que permitiria uma comparação e conclusões mais precisas dos resultados.

Ao realizar um estudo com mulheres que realizaram cirurgia para neoplasia da mama é relatado que existe uma diferença na PImáx e na PEmáx no pré-operatório se comparado com o pós-operatório imediato e tardio. Também houve diferença nas variáveis VEF1 e CVF, demonstrando que há uma redução no pós-operatório comparado com o pré-operatório, porém os valores retornam aos apresentados no pré-operatório dentro de trinta dias. Discordando deste estudo, pois, os valores não retornaram completamente aos do pré-operatório dentro de um mês ${ }^{10}$. A capacidade vital só reduz quando há uma diminuição da força dos músculos respiratórios de pelo menos $50 \%$ do vaIor previsto. Embora, somente tenha sido avaliado 
a CVF, o comportamento da função pulmonar foi muito semelhante ao encontrado para as forças musculares respiratórias².

A redução dos valores da função pulmonar e da força muscular respiratória pode ser devido à presença do dreno, movimentos respiratórios superficiais, receio de sentir dor e a utilização de analgésicos ${ }^{10}$. Todas essas variáveis podem causar diminuição dos volumes pulmonares e consequentemente complicações pulmonares, que podem ser prevenidas ou minimizadas com a inserção de um programa elaborado precocemente de fisioterapia. Esta afirmação concorda com este estudo, pois a realização da fisioterapia imediata pode ter contribuído para o retorno dos valores ao esperado.

Não foi encontrado na literatura revisada publicações científicas que trazem a aplicação de exercícios com os membros superiores em mulheres mastectomizadas, com o objetivo de observar se há alterações na função pulmonar e da força muscular respiratória.

Os exercícios fisioterapêuticos precoces podem ter colaborado para o retorno aos valores previstos de força muscular respiratória e da função pulmonar, no entanto não foram capazes de retornar completamente aos valores no pré-operatório. Isto pode ter ocorrido devido ao número reduzido de sessões de fisioterapia, ou seja, necessita-se de um programa de fisioterapia com maior duração.

Tendo em vista não somente a recuperação do câncer, mas também a reabilitação global no âmbito físico, a fisioterapia torna-se imprescindível para a vida da mulher, pois além de oferecer técnicas terapêuticas físicas passíveis de intervir desde a mais precoce recuperação funcional, até a profilaxia das sequelas, além de reduzir o tempo de recuperação, com retorno mais rápido às atividades cotidianas, colaborando com sua reintegração à sociedade 5 .

Algumas limitações deste estudo devem ser citadas, a saber: Não podemos afirmar que a diminuição da dor, a normalização para os valores previstos da PImáx, PEmáx e da função pulmonar deuse devido ao protocolo de exercícios propostos ou se estes valores retornaram aos esperados devido ao reestabelecimento das funções do próprio organismo. Contudo, faz-se necessário a realização de um grupo controle, ou seja, sem intervenção fisioterapêutica como forma de comparar se essas variáveis alteram devido aos exercícios cinesioterapêuticos.

\section{Considerações finais}

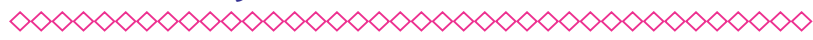

Os exercícios contribuíram para a normalização dos parâmetros respiratórios analisados (força muscular respiratória e função pulmonar) e melhora importante da dor no pós-operatório, contudo, eles não foram capazes de retornar os parâmetros respiratórios aos encontrados no momento pré-operatório.

Todavia, embora este trabalho se configure em um estudo de caso, entendemos ser importante um tratamento fisioterapêutico precoce em muIheres que realizaram mastectomia, para contribuir na recuperação mais rápida do quadro álgico e respiratório.

\section{Referências}

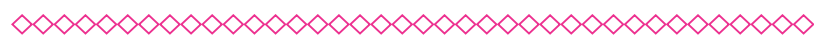

1. Inumaru LE, Silveira EA, Naves MM. Fatores de risco e de proteção para câncer de mama: uma revisão sistemática. Cad Saúde Pública. 2011; 27(7):1259-70.

2. Schettino RC, Jotta LMGN, Cassali GD. Função pulmonar em mulheres com câncer de mama submetidas à radioterapia: um estudo piloto. Fisioter Pesqui. 2010; 17(3):248-52.

3. Nascimento SL, Oliveira RR, Oliveira MMF, Amaral MTF. Complicações e condutas fisioterapêuticas após cirurgia por câncer de mama: estudo retrospectivo. Fisioter Pesqui. 2012;19(3):248-255.

4. Pereira CAC, Neder JA. Diretrizes para testes de função pulmonar. J Bras Pneumol. 2002;28(3):1-238.

5. Camargo MC, Marx AG. Reabilitação Física no Câncer de Mama. São Paulo: Roca; 2000.

6. Neder JA, Andreoni S, Lerario MC, Nery LE. Reference values for lung function tests. II. Maximal respiratory pressures and voluntary ventilation. Braz J Med Biol Res. 1999;32(6):719-27. 
7. Leal TO, Cardoso KQ, Kalif SK, Almeida FB, Fontelles MJ. A Fisioterapia no Linfedema Pós-mastectomia a Madden. Rev Para Med. 2004;18(1):42-5

8. Costa AIS, Chaves MD. Dor em pacientes oncológicos sob tratamento quimioterápico. Rev Dor. 2012;13(1):45-9.

9. Zanon DS, Abreu DL, Braz MM. Avaliação do padrão respiratório em mulheres submetidas a cirurgias para tratamento do câncer de mama. In: XV Simpósio de Ensino, Pesquisa e Extensão. Santa Maria; 2011.

10. Bregagnol RK, Dias AS. Alterações funcionais em mulheres submetidas à cirurgia de mama com linfadenectomia axilar total. Rev Bras Cancerol. 2010;56(1):25-33.

11. Pacheco $M N$, Filho AD, Melo DAS. Fisioterapia para o tratamento do linfedema no pós-operatório de mastectomia: revisão de literatura. Rev Fac Cienc Med Sorocaba. 2011;13(4):4-7.

12. Picaró P, Perloiro FA. Evidência da Intervenção Precoce da Fisioterapia em Mulheres Mastectomizadas: Estudo Comparativo. EssFisiOnline. 2005 Mar;1(2):3-14.

13. Rezende LF, Franco RL, Gurgel MSC. Fisioterapia aplicada à fase pós-operatória de câncer de mama: o que considerar. Rev Cienc Med. 2005;14(3):295-302.

14. Onaga FI; Jamami M, Ruas G, Lorenzo VAP, Jamami LK. Influência de diferentes tipos de bocais e diâmetros de traqueias na manovacuometria. Fisioter Mov. 2010;23(2):211-19.

15. Bellinetti LM, Thomson JC. Avaliação muscular respiratória nas toracotomias e laparotomias superiores eletivas. J Bras Pneumol. 2006;32(2)99-105. 


\section{Anexos}

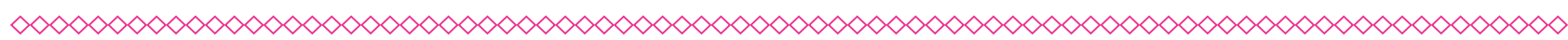

Figura 1 - Percepção da dor no pré e pós-operatório e no decorrer das 10 sessões de fisioterapia, 2016

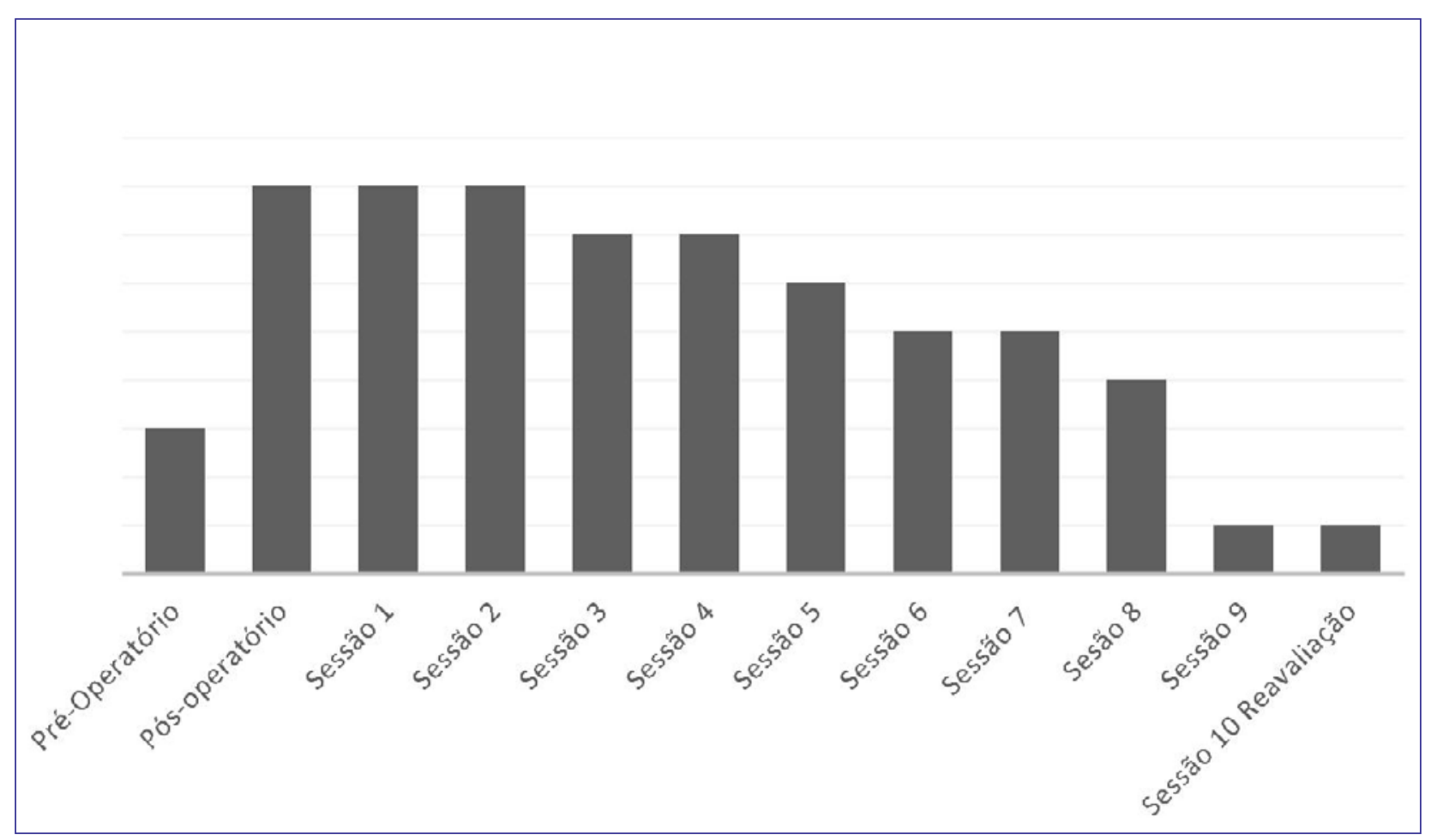

Fonte: Elaborado pelos autores

(clique para voltar ao texto)

Tabela 1 - Força da musculatura respiratória no pré e pós-operatório e após as 10 sessões de fisioterapia, 2016

\begin{tabular}{lcccc} 
& $\begin{array}{c}\text { Pré- } \\
\text { operatório }\end{array}$ & $\begin{array}{c}\text { Pós- } \\
\text { operatório }\end{array}$ & $\begin{array}{c}\text { Pós- } \\
\text { atendimento }\end{array}$ & $\begin{array}{c}\text { Valores } \\
\text { Previstos }\end{array}$ \\
$\begin{array}{l}\text { PImáx } \\
(\mathrm{cmH2O})\end{array}$ & -100 & -60 & -84 & -81 \\
\hline $\begin{array}{l}\text { PEmáx } \\
(\mathrm{cmH} 2 \mathrm{O})\end{array}$ & 80 & 60 & 82 & 79 \\
\hline
\end{tabular}

*PImáx= força muscular respiratória inspiratória máxima, PEmáx= força muscular respiratória expiratória. Fonte: Elaborado pelos autores 
Tabela 2 - Valores da função pulmonar no pré e pós-operatório e após as 10 sessões de fisioterapia, 2016

\begin{tabular}{lcccc} 
& $\begin{array}{c}\text { Pré- } \\
\text { operatório }\end{array}$ & $\begin{array}{c}\text { Pós- }^{-} \\
\text {operatório }\end{array}$ & $\begin{array}{c}\text { Pós- } \\
\text { atendimento }\end{array}$ & $\begin{array}{c}\text { Valores } \\
\text { Previstos }\end{array}$ \\
CVF/L & 3,48 & 2,84 & 3,02 & 3,19 \\
VEF1/ L & 3,05 & 2,66 & 2,89 & 2,55 \\
PFE/ L & 6,67 & 5,84 & 8,38 & 5,95 \\
FEF 25/75 & 4,44 & 4,35 & 4,62 & 2,56 \\
FEV1\% & 87,6 & 93,7 & 95,7 & 79,4 \\
\hline
\end{tabular}

$\mathrm{CVF}=$ capacidade vital forçada, $\mathrm{VEF} 1=$ volume expiratório forçado no primeiro segundo, PFE= pico de fluxo expiratório, FEF 25/75= fluxo expiratório forçado, FEV1\%= razão VEF1/CVF, L=Litro.

Fonte: Elaborado pelos autores 